Conclusion* Assessment of the residual fatty tissue results in the finding of $30 \%$ more lymph nodes. This did not result in the detection of more lymph node metastases. We do think it is worthwhile to investigate the residual fatty tissue in women with a high $\mathrm{BMI}(>30 \mathrm{~kg} / \mathrm{m} 2)$.

\section{GENOMIC ALTERATION OF A METASTATIC GASTRIC- TYPE CERVICAL ADENOCARCINOMA: HINTS AND PERSONALIZED TREATMENT OPTIONS}

${ }^{1} \mathrm{~A}$ Sarivalasis, ${ }^{2} \mathrm{~B}$ Bisig, ${ }^{3} \mathrm{D}$ Hastir, ${ }^{3} \mathrm{~K}$ Lefort, ${ }^{1} \mathrm{~A}$ Liapi*. 'Lausanne university hospital, Medical Oncology, Lausanne, Switzerland; 'Lausanne university hospital, Clinical Pathology, Lausanne, Switzerland; 'ausanne, Clinical Pathology, Lausanne, Switzerland

\subsection{6/ijgc-2021-ESGO.524}

Introduction/Background* Cervical adenocarcinoma accounts for $20-25 \%$ of all uterine cervical cancers with $90 \%$ of cases being associated with HPV. Of the remaining, non-HPV associated adenocarcinomas, the most common subtype is the gastric-type (GAS). GAS portends a poorer prognosis irrespective of tumor stage. We report the genomic study of a stage FIGO IVB GAS.

Methodology A 52 years old patient was admitted for atypical glandular cells (AGC) on the cervical smear. Pelvic MRI described a mass infiltrating the proximal third of the vagina, the cervix and the isthmus of the uterus and suggested paraaortic lymphadenopathies. The PET-CT confirmed the nodal involvement and a right iliac bone lesion. Both cervical and bone biopsies were positive for a p53 positive, p16, HER2 and PD-L1 (SP263 clone) negative, GAS. In the absence of standard of care treatment we performed a 52-gene next generation sequencing (NGS) of the cervical biopsies, containing $30 \%$ tumor cells

Result(s)* NGS detected the following pathogenic mutations: TP53 exon 8 (p.Arg280Thr, variant allele frequency (VAF) 18\%), ERBB2 exon 20 (p.Val777Leu, VAF 14\%), KRAS exons 2 (p.Gly12Ser, VAF 4\%) and 4 (p.Ala146Val, VAF 5\%).

Conclusion* The most frequently mutations reported in GAS are TP53, STK11, and CDKN2A. The presence of a generally, mutually exclusive, ERBB2 and KRAS in our patient is a rare constellation potentially suggestive of multiple subclones. In the absence of a specific treatment guideline for GAS we initiated a cisplatin, paclitaxel and bevacizumab treatment combination. Based on the NGS results, in case of treatment failure, a MAPK targeted treatment by a MEK inhibitor could be considered.

\section{CLINICOPATHOLOGICAL FEATURES OF PATIENTS WITH ENDOMETRIAL STROMAL NODULE AND ENDOMETRIAL STROMAL SARCOMA, SINGLE CENTER EXPERIENCE}

${ }^{1} \mathrm{M}$ Emiroglu, ${ }^{1} \mathrm{CK}$ Talu, ${ }^{2} \mathrm{Z}$ Guzeloz, ${ }^{3} \mathrm{HI}$ Yildiz, ${ }^{1} \mathrm{D}$ Ayaz, ${ }^{4} \mathrm{M}$ Gokcu*. ${ }^{1}$ University of Health Sciences, Tepecik Training and Research Hospital, pathology: ${ }^{2}$ University of Health Sciences, Tepecik Training and Research Hospital, Radiation Oncology, Turkey; ${ }^{3}$ University of Health Sciences, Tepecik Training and Research Hospital, Medical Oncology, Turkey; ${ }^{4}$ IEÜ Medical Park Hastanesi, Gynecological Oncology

\subsection{6/ijgc-2021-ESG0.525}

Introduction/Background* To present the clinicopathological features of cases with Endometrial Stromal Nodule (ESN) and Endometrial Stromal Sarcoma (ESS) in our center.
Methodology The cases diagnosed between 2008-2020 were re-examined microscopically. The information for clinical follow-up were retrieved from the hospital archives, retrospectively.

Result(s)* ESN cases consisted of 11 patients between the ages of 41-68 (mean 51,8), Low Grade Endometrial Stromal Sarcoma (LGESS) cases consisted of 13 patients between the ages of 24-53 (mean 44), High Grade Endometrial Stromal Sarcoma (HGESS) cases consisted of 9 patients between the ages of 60-84 (mean 71,2).

Mean tumor size was $3,3 \mathrm{~cm}(1,7-8 \mathrm{~cm})$ in ESN cases; 6,4 $\mathrm{cm}(1-13 \mathrm{~cm})$ in LGESS cases; $6,5 \mathrm{~cm}(4,5-9 \mathrm{~cm})$ in HGESS cases.

In LGESS cases, the number of mitosis in 10 high power fields was 0-7 (average 2,8); In HGESS cases it was counted as $10-45$ (average 22). Ki-67 proliferation index was evaluated as $2-5 \%$ (mean 3.8\%) in LGESS cases; as 30-100\% (mean $68 \%)$ in HGESS cases.

At the median follow-up of 56,5 months (range, 21-147 months), all of 11 ESN cases are alive. At the median followup of 60 months (range, 47-144 months), 1 of 13 case of LGESS have died (\%8). At the median follow-up of 8 months (range, 1-53 months), 5 of 9 cases of HGESS have died (\% 56). Distant metastasis was seen only in 1 case of 9 HGESS, the location of metastasis is lung.

$85 \%$ of cases with LGESS were presented in stage I and,\% 15 were in stage II. $37.5 \%$ of cases with HGESS were presented in stage I, 50\% were in stage III and $12.5 \%$ were in stage IV.

Conclusion* Endometrial stromal neoplasms are rare lesions of the uterus, usually presenting in the postmenopausal period. The histological type and stage of these lesions are directly related to the clinical course. Average mitotic activity and Ki67 levels can also provide information in the direction of the clinical course.

\section{RELIABLE IDENTIFICATION OF ENDOMETRIAL PRECANCERS THROUGH PAX2, $\beta$-CATENIN, AND PTEN IMMUNOHISTOCHEMISTRY}

D Castrillon*, M Aguilar. UT Southwestern Medical Center, Dallas, USA

\subsection{6/ijgc-2021-ESG0.526}

Introduction/Background* The diagnosis of endometrial atypical hyperplasia/endometrioid intraepithelial neoplasia ( $\mathrm{AH} /$ EIN) remains challenging and subjective in some cases, with variable histologic criteria and differences of opinion among gynecologic pathologists, potentially leading to under/overtreatment. There has been growing interest in the use of specific immunohistochemical markers as adjuncts in AH/EIN diagnosis. For example, the WHO 2020 Classification lists loss of Pten, Pax2, or mismatch repair proteins as desirable diagnostic criteria. Other markers, most notably b-catenin and Arid1a, are also aberrantly expressed in some AH/EIN. However, the performance of these markers individually- and more importantly as a group - has not been rigorously explored, raising critical questions as to which marker(s) or combination(s) thereof is the most efficient and reliable in practice.

Methodology Inclusion criteria was a diagnosis of $\mathrm{AH} / \mathrm{EIN}$ on an endometrial tissue sample based on histologic features. Formalin-fixed/paraffin-embedded tissue sections from $n=111$ 